\title{
A CLASSIFICATION OF SIMPLE LIE MODULES HAVING A 1-DIMENSIONAL WEIGHT SPACE
}

\author{
D. J. BRITTEN AND F. W. LEMIRE
}

\begin{abstract}
Let $L$ denote a simple Lie algebra over the complex numbers. In this paper, we classify and construct all simple $L$ modules which may be infinite dimensional but have at least one 1-dimensional weight space. This completes the study begun earlier by the authors for the case of $L=A_{n}$. The approach presented here relies heavily on the results of Suren Fernando whose dissertation dealt with simple weight modules and their weight systems.
\end{abstract}

0. Introduction. Let $L$ be a finite-dimensional simple Lie algebra over the complex field $\mathrm{C}$ having a Cartan subalgebra $H$ and denote by $C(L)$ the centralizer of $H$ in the universal enveloping algebra $U$ of $L$. If $\lambda: H \rightarrow \mathrm{C}$ is a weight function of a 1-dimensional weight space $M_{\lambda}$ in a simple $L$ module $M$ then $\eta: C(L) \rightarrow \mathbf{C}$, defined by $\eta(c) v=c v$ for $v \in M_{\lambda}$ and $c \in C(L)$, is an algebra homomorphism called a mass function of $M$. Clearly $\eta$ restricted to $H$ is equal to $\lambda$. Conversely, given any algebra homomorphism $\eta: C(L) \rightarrow \mathrm{C}$ one can construct a unique simple $L$ module which admits $\eta$ as a mass function $[4,10]$. In $[4]$ the authors determined all algebra homomorphisms $\eta: C(L) \rightarrow \mathrm{C}$ for the simple Lie algebras $L$ of type $A_{n}$ and using these classified all "pointed" $A_{n}$ modules (where we call a module pointed if it is simple and has at least one 1-dimensional weight space). In this paper we complete the classification of all pointed $L$ modules for arbitrary simple Lie algebras. The collection of all pointed $L$ modules clearly includes the highest weight $L$ modules and is included in the collection of all Harish-Chandra $L$ modules relative to the Cartan subalgebra $H$ (-i.e. simple $L$ modules having a weight space decomposition and finite-dimensional weight spaces relative to $H$ ). The latter inclusion is strict since there exist examples of Harish-Chandra $A_{2}$ modules in which every weight space is two dimensional. In the special case of $A_{1}$ modules, every Harish-Chandra $A_{1}$ module is pointed.

Our approach to this problem makes heavy use of the results from Fernando's thesis [8]. In particular, from Fernando's results we see that in place of determining all algebra homomorphisms $\eta: C(L) \rightarrow \mathrm{C}$ it suffices to find only those algebra homomorphisms $\eta$ which are associated with so-called pointed "torsion free" $L$

Received by the editors May 23, 1984. This paper was presented at the 1984 Canadian Mathematical Society's Summer Seminar on Lie algebras and related topics held at the University of Windsor.

1980 Mathematics Subject Classification. Primary 17B10.

Key words and phrases. Simple Lie modules, weight space decomposition, torsion free module.

Research partially supported by NSERC operating grants \#A8471 and \# A7742. 
modules (these are defined in $\S 1$ ) and further that such modules can only occur for simple Lie algebras of type $A_{n}$ or $C_{n}$.

In $\S 1$ we outline the relevant results from Fernando's thesis and other background material which will be used in this paper. $\$ 2$ contains the construction of canonical examples of pointed torsion free $A_{n}$ and $C_{n}$ modules. The main results of this paper are contained in $\S \S 3$ and 4 where we show that the examples given in $\S 2$ exhaust all pointed torsion free $A_{n}$ and $C_{n}$ modules and hence by Fernando's results exhaust all such modules. Finally in $\S 5$ we provide an alternate construction of all pointed $L$ modules by constructing a mass function associated with each one. This allows us to realize all pointed $L$ modules directly as quotient modules of the universal enveloping algebra $U(L)$.

1. Structure of simple weight modules. In this section, we review the results of Fernando [8] on simple weight modules, and establish some basic facts about pointed modules. Fernando's results reduce the classification of pointed modules of simple Lie algebras to the classification of pointed, torsion free modules of the simple Lie algebras of types $A$ and $C$.

Throughout this section $L$ denotes a reductive Lie algebra, $H$ a Cartan subalgebra of $L, R$ the root system of $(L, H), B$ a base of $R, R_{B}^{+}$the positive roots relative to $B$, and for $B_{1} \subseteq B,\left\langle B_{1}\right\rangle$ denotes the integer linear span of $B_{1}$ intersected with $R$. Also, we express the root space decomposition of $L$ by $L=H \oplus \sum_{\alpha \in R} \oplus L_{\alpha}$, and denote a root vector of $L$ belonging to $\alpha \in R$ by $X_{\alpha}$. For notational convenience, occasionally we use $Y_{\alpha}$ to denote $X_{-\alpha}$.

DeFinition 1.1. $M$ is an $(L, H)$ weight module provided

(i) $M$ is a finitely generated $L$ module, and

(ii) $M=\sum_{\lambda \in H^{*}} \oplus M_{\lambda}$ where for $\lambda \in H^{*}, M_{\lambda}=\{v \in M \mid h v=\lambda(h) v$ for all $h \in H\}$ is finite dimensional.

Definition 1.2. Let $M$ be an $(L, H)$ weight module. $M$ is torsion free provided the action of $X_{\alpha}$ on $M, X_{\alpha}: M \rightarrow M$, is injective for all $\alpha \in R . M$ is said to be pointed provided it is simple and has a 1-dimensional weight space.

THEOREM 1.3 [FERNANDO]. If $L$ is a finite-dimensional complex reductive Lie algebra which admits a nonzero, torsion free weight module then the simple ideals of $L$ are of two possible types; type $A$ and type $C$.

Let $M$ be an arbitrary simple $(L, H)$ weight module. Let $T(M)$ be the set of all roots $\alpha \in R$ such that $X_{\alpha}: M \rightarrow M$ is injective. As shown in [8], for some base $B$ of $R$ and some subset $B_{1}$ of $B$ the parabolic subset $P=\left\langle B_{1}\right\rangle \cup R_{B}^{+}$is related to $T(M)$ by the following two properties:

(i) $P \cap(-P)=T(M) \cap(-T(M))$, and

(ii) $T(M) \cap(R \backslash-T(M)) \subseteq P \cap(R \backslash-P)$.

Setting $P_{s}=P \cap(-P)$ and $P_{a}=P \cap(R \backslash-P)$, we define the following subalgebras of $L$ :

$$
p^{-}=H \oplus \sum_{\alpha \in P} \oplus L_{\alpha}, \quad g=H \oplus \sum_{\alpha \in P_{s}} \oplus L_{\alpha}, \quad u^{-}=\sum_{\alpha \in P_{a}} \oplus L_{\alpha}
$$




$$
u^{+}=\sum_{\alpha \in-P_{a}} \oplus L_{\alpha}, \quad p^{+}=g \oplus u^{+}
$$

Definition 1.4. $M^{u^{+}}=\left\{v \in M \mid u^{+} v=0\right\}$.

THEOREM 1.5 [FERNANDo]. Let $W(M)$ denote the set of weights of $M$ and $\mathbf{Z}$ the set of integers. If $\alpha \in R$ and $\lambda \in W(M)$ then $(\lambda+\mathbf{Z} \alpha) \cap W(M)$ is an interval and $X_{\alpha}$ is locally nilpotent if and only if $\left(\lambda+\mathbf{Z}^{+} \alpha\right) \cap W(M)$ is finite. Moreover, if $X_{\alpha}$ is not locally nilpotent then it is torsion free.

TheOREM 1.6 [FERNANDO]. $M^{u^{+}}$is a nonzero simple, torsion free $(g, H)$ weight module. Moreover, $M^{u^{+}}$is equal to a sum of complete weight spaces of $M$.

THEOREM 1.7 [FERNANDO]. $M$ is equivalent to the unique simple quotient of the $(L, H)$ weight module $U(L) \otimes_{U\left(p^{+}\right)} M^{u^{+}}$.

We close this section with an outline of some basic results on pointed, torsion free $L$ modules $M$ which are used in the subsequent sections of this paper. In particular, it is clear that all nonzero weight spaces of $M$ are 1 dimensional and for $\mu_{i} \in R$ the monomial $X_{\mu_{1}} \cdots X_{\mu_{k}}$ acts bijectively on $M$ mapping $M_{\lambda}$ onto $M_{\lambda+\mu_{1}+\cdots+\mu_{k}}$. Therefore, it follows that if $\mu, \nu, \mu+\nu \in R$ and $M_{\lambda}$ is a nonzero weight space of $M$, there exists a nonzero scalar $K_{\lambda}(\mu, \nu)$ such that $\left(X_{\mu} X_{\nu}-K_{\lambda}(\mu, \nu) X_{\mu+\nu}\right) M_{\lambda}=\{0\}$. If $C(L)$ denotes the cycle subalgebra of $U(L)$, i.e. the centralizer of the Cartan subalgebra $H$ in the universal enveloping algebra $U(L)$ of $L$, then each weight function $\lambda \in H^{*}$ of $M$ can be extended uniquely to an algebra homomorphism $\eta$ : $C(L) \rightarrow \mathrm{C}$ referred to as a mass function of $M$. Its action is defined by $c v_{\lambda}=\eta(c) v_{\lambda}$ for all $c \in C(L)$. Evidently, the existence of this algebra homomorphism requires only that $\operatorname{dim} M_{\lambda}=1$ and not that $M$ is torsion free.

THEOREM 1.8 [4, 10]. A pointed module $M$ is determined, up to equivalence, by any of its mass functions.

In the case of pointed, torsion free modules we can strengthen this result to read

THEOREM 1.9. Let $M_{1}$ and $M_{2}$ be pointed, torsion free $L$ modules admitting $a$ common weight function $\lambda$. Let $\eta_{1}$ and $\eta_{2}$ be mass functions for $M_{1}$ and $M_{2}$ with respect to $\lambda$ such that $\eta_{1}(c)=\eta_{2}(c)$ for all monomials $c \in C(L)$ of degree less than or equal to three. Then $M_{1}$ is equivalent to $M_{2}$.

Proof. Let $\left(M_{1}\right)_{\lambda}=\mathbf{C} v_{1}$ and $\left(M_{2}\right)_{\lambda}=\mathbf{C} v_{2}$. As previously pointed out, if $\mu, \nu$, $\mu+\nu \in R$, then there are nonzero complex numbers $K_{1}$ and $K_{2}$ such that

$$
\left(X_{\mu} X_{\nu}-K_{1} X_{\mu+\nu}\right) v_{1}=0
$$

and

$$
\left(X_{\mu} X_{\nu}-K_{2} X_{\mu+\nu}\right) v_{2}=0
$$


Since

$$
\eta_{1}\left(X_{-(\mu+\nu)} X_{\mu} X_{\nu}\right)=\eta_{2}\left(X_{-(\mu+\nu)} X_{\mu} X_{\nu}\right)
$$

and

$$
\eta_{1}\left(X_{-(\mu+\nu)} X_{\mu+\nu}\right)=\eta_{2}\left(X_{-(\mu+\nu)} X_{\mu+\nu}\right)
$$

we know that $K_{1}=K_{2}$.

Let $c=z_{1} \cdots z_{k}$ be a monomial of degree $k$ in the Poincaré-Birkhoff-Witt basis which is in $C(L)$. We prove $\eta_{1}(c)=\eta_{2}(c)$ by induction on $k$. This is true for $k \leqslant 3$ by assumption. The induction assumption, that $\eta_{1}(c)=\eta_{2}(c)$ for all $c$ of degree less than $k$, permits us to permute the $z_{i}$ 's since such a permutation is done at the expense of adding lower degree terms. Either $c$ is an element in the universal enveloping algebra of $H$ in which case the result is clear or

$$
z_{1} \cdots z_{k}=X_{\mu_{1}} \cdots X_{\mu_{k-2}} X_{\mu} X_{\nu}+\text { polynomial of degree }<k
$$

where $\mu_{1}, \ldots, \mu_{k-2}, \mu, \nu, \mu+\nu \in R$. Our induction assumption implies we need only show that

$$
\eta_{1}\left(X_{\mu_{1}} \cdots X_{\mu_{k-2}} X_{\mu} X_{\nu}\right)=\eta_{2}\left(X_{\mu_{1}} \cdots X_{\mu_{k-2}} X_{\mu} X_{\nu}\right)
$$

But for $i=1,2$ we know that

$$
\begin{aligned}
\eta_{i}\left(X_{\mu_{1}} \cdots X_{\mu_{k-2}} X_{\mu} X_{\nu}\right) v_{i} & =X_{\mu_{1}} \cdots X_{\mu_{k-2}} X_{\mu} X_{\nu} v_{i}=K_{i} X_{\mu_{1}} \cdots X_{\mu_{k-2}} X_{\mu+\nu} v_{i} \\
& =K_{i} \eta_{i}\left(X_{\mu_{1}} \cdots X_{\mu_{k-2}} X_{\mu+\nu}\right) v_{i} .
\end{aligned}
$$

Therefore,

$$
\begin{aligned}
\eta_{1}\left(X_{\mu_{1}} \cdots X_{\mu_{k-2}} X_{\mu} X_{\nu}\right) & =K_{1} \eta_{1}\left(X_{\mu_{1}} \cdots X_{\mu_{k-2}} X_{\mu+\nu}\right)=K_{2} \eta_{2}\left(X_{\mu_{1}} \cdots X_{\mu_{k-2}} X_{\mu+\nu}\right) \\
& =\eta_{2}\left(X_{\mu_{1}} \cdots X_{\mu_{k-2}} X_{\mu} X_{\nu}\right) .
\end{aligned}
$$

2. Construction of simple torsion free modules. In this section, we construct certain simple, torsion free modules of the Lie algebras of types $A$ and $C$ which we show later exhaust all such modules. These are slight variants of those presented by Fernando in [8].

The Weyl algebra $W_{n}$ of order $n$ can be realized as the associative subalgebra of End ${ }_{\mathbf{C}} \mathbf{C}\left[x_{1}, \ldots, x_{n}\right]$ generated by $\left\{x_{i}, \delta_{i} \mid i=1, \ldots, n\right\}$ where $x_{i}$ and $\delta_{i}$ are viewed as left multiplication by $x_{i}$ and partial differentiation with respect to $x_{i}$, respectively. Following [6, Chapter 4.6] the simple Lie algebra $C_{n}$ can be embedded in the subalgebra of degree two elements of $W_{n}$ as indicated below.

The root system $R$ of $C_{n}$ can be identified with the set of vectors

$$
\left\{ \pm\left(\varepsilon_{i} \pm \varepsilon_{j}\right) \mid 1 \leqslant i<j \leqslant n\right\} \cup\left\{ \pm 2 \varepsilon_{i} \mid i=1, \ldots, n\right\}
$$

in $\mathbf{R}^{n}$ where $\varepsilon_{i}$ denotes the $i$ th standard basis vector of $\mathbf{R}^{n}$. A base $B$ for $R$ is given by $B=\left\{\varepsilon_{1}-\varepsilon_{2}, \ldots, \varepsilon_{n-1}-\varepsilon_{n}, 2 \varepsilon_{n}\right\}$. For computational purposes throughout this paper, we fix a Chevalley basis of $C_{n}$ which we call standard. This is one of the form

$$
\left\{X_{ \pm\left(\varepsilon_{1} \pm \varepsilon_{1}\right)} \mid 1 \leqslant i<j \leqslant n\right\} \cup\left\{X_{ \pm 2 \varepsilon_{l}} \mid i=1, \ldots, n\right\} \cup\left\{h_{\alpha_{l}} \mid \alpha_{i} \in B\right\}
$$


having the property that the map $\phi: C_{n} \rightarrow W_{n}$ given by

$$
\begin{array}{ll}
\phi\left(X_{\varepsilon_{i}-\varepsilon_{j}}\right)=x_{i} \delta_{j} & \text { for } 1 \leqslant i \neq j \leqslant n, \\
\phi\left(X_{\varepsilon_{i}+\varepsilon_{j}}\right)=x_{i} x_{j} & \text { for } i, j=1, \ldots, n, \\
\phi\left(X_{-\left(\varepsilon_{i}+\varepsilon_{j}\right)}\right)=\delta_{i} \delta_{j} & \text { for } i, j=1, \ldots, n, \\
\phi\left(h_{\varepsilon_{i}-\varepsilon_{i+1}}\right)=x_{i} \delta_{i}-x_{i+1} \delta_{i+1} & \text { for } i=1, \ldots, n-1, \\
\phi\left(h_{2 \varepsilon_{n}}\right)=\left(x_{n} \delta_{n}+\delta_{n} x_{n}\right) / 2 &
\end{array}
$$

is a Lie algebra isomorphism.

A modification of the $C_{n}$ module $\mathbf{C}\left[x_{1}, \ldots, x_{n}\right]$ produces the module $M(a)$ which we seek. For each $n$-tuple $a=\left(a_{1}, \ldots, a_{n}\right) \in \mathbf{C}^{n}$ with $a_{i} \notin \mathbf{Z}$ for all $i$, we define $M(a)$ to be the complex linear space spanned by

$$
\left\{x^{b}=x_{1}^{b_{1}} \cdots x_{n}^{b_{n}} \mid b_{i}-a_{i} \in \mathbf{Z} \text { for all } i \text { and } \sum_{i=1}^{n}\left(b_{i}-a_{i}\right) \in 2 \mathbf{Z}\right\} .
$$

The linear space $M(a)$ can be viewed as a $C_{n}$ module through the natural action of $\phi\left(C_{n}\right)$ on $M(a)$. One can easily show that distinct $x^{b} \in M(a)$ form a basis for distinct weight spaces for $M(a)$. The $C_{n}$ module $M(a)$ is torsion free, since

$$
\begin{array}{ll}
X_{\varepsilon_{i}-\varepsilon_{j}} x^{b}=b_{j} x^{b+\varepsilon_{i}-\varepsilon_{j}} & \text { for } 1 \leqslant i \neq j \leqslant n, \\
X_{\varepsilon_{i}+\varepsilon_{j}} x^{b}=x^{b+\varepsilon_{i}+\varepsilon_{j}} & \text { for } i, j=1, \ldots, n, \\
X_{-\left(\varepsilon_{i}+\varepsilon_{j}\right)} x^{b}=b_{i} b_{j} x^{b-\varepsilon_{i}-\varepsilon_{j}} & \text { for } i \neq j=1, \ldots, n, \\
X_{-2 \varepsilon_{i}} x^{b}=b_{i}\left(b_{i}-1\right) x^{b-2 \varepsilon_{i}} & \text { for } i=1, \ldots, n,
\end{array}
$$

and none of the $b_{i}$ 's are integers.

The following argument shows that $M(a)$ is a simple $C_{n}$ module. For $x^{b}$, $x^{c} \in M(a)$ we have $b-c=\sum_{i=1}^{n} k_{i} \varepsilon_{i}$ where $k_{i} \in \mathbf{Z}$ and $\sum_{i=1}^{n} k_{i} \in 2 \mathbf{Z}$. Therefore,

$$
\begin{aligned}
\sum_{i=1}^{n} k_{i} \varepsilon_{i}= & k_{1}\left(\varepsilon_{1}-\varepsilon_{2}\right)+\left(k_{1}+k_{2}\right)\left(\varepsilon_{2}-\varepsilon_{3}\right) \\
& +\cdots+\left(\sum_{i=1}^{n-1} k_{i}\right)\left(\varepsilon_{n-1}-\varepsilon_{n}\right)+\left(\frac{1}{2}\right)\left(\sum_{i=1}^{n} k_{i}\right) 2 \varepsilon_{n}
\end{aligned}
$$

so that

$$
X_{s_{1}\left(\varepsilon_{1}-\varepsilon_{2}\right)}^{e_{1}} \cdots X_{s_{n}\left(2 \varepsilon_{n}\right)}^{e_{n}} x^{c}
$$

is a nonzero multiple of $x^{b}$ where $e_{i}=\left|\sum_{j=1}^{i} k_{j}\right|$ for $i=1, \ldots, n-1$ and $e_{n}$ $=\frac{1}{2}\left|\sum_{j=1}^{n} k_{j}\right|$ and $s_{i}=\operatorname{sgn} \sum_{j=1}^{i} k_{j}$. Summarizing these results we have

THEOREM 2.1. For each $n$-tuple $a=\left(a_{1}, \ldots, a_{n}\right) \in \mathbf{C}^{n}$ with $a_{i} \notin \mathbf{Z}$ for all $i, M(a)$ is a pointed, torsion free $C_{n}$ module.

The root system $R$ of $C_{n}$ contains a subsystem $\left\{ \pm\left(\varepsilon_{i}-\varepsilon_{j}\right) \mid 1 \leqslant i<j \leqslant n\right\}$ which is equivalent to a root system of the simple Lie algebra $A_{n-1}$. The basal vectors in a standard base of $C_{n}$ corresponding to this subsystem form a base for $A_{n-1}$ which we 
call a standard base for $A_{n-1}$. When discussing simple Lie algebras of type $A$, we use this base. For each $n$-tuple $a=\left(a_{1}, \ldots, a_{n}\right) \in \mathbf{C}^{n}$ with $a_{i} \notin \mathbf{Z}$ for all $i, N(a)$ is defined to be the complex linear space spanned by

$$
\left\{x^{b}=x_{1}^{b_{1}} \cdots x_{n}^{b_{n}} \mid b_{i}-a_{i} \in \mathbf{Z} \text { for all } i \text { and } \sum_{i=1}^{n}\left(b_{i}-a_{i}\right)=0\right\} \text {. }
$$

$N(a)$ can be viewed as an $A_{n-1}$ module through the natural action of $\phi\left(A_{n-1}\right)$ on $N(a)$. By an analysis similar to that described for the previous case, we have

THEOREM 2.2. For each $n$-tuple $a=\left(a_{1}, \ldots, a_{n}\right) \in \mathbf{C}^{n}$ with $a_{i} \notin \mathbf{Z}$ for all $i, N(a)$ is a pointed, torsion free $A_{n-1}$ module.

3. Pointed, torsion free $A_{n}$ modules. As indicated by Theorem 1.8 , the determination of pointed modules can be achieved by determining the mass functions defined on $C(L)$. In [4], the authors constructed all possible mass functions on $C\left(A_{n}\right)$. In this section, we show that the mass functions of the pointed torsion free $A_{n}$ modules of the form $N(a)$ constructed in $\S 2$ exhaust all mass functions of pointed torsion $A_{n}$ modules. It follows that every pointed torsion free $A_{n}$ module is equivalent to one of the form $N(a)$.

First, we treat the case of $M$ being an $A_{2}$ module. Let $\{\alpha, \beta\}$ be a base for the root system of $A_{2}$. Let $M_{\lambda}=\mathrm{C} v_{\lambda}$ be a weight space of $M$. The basic cycles of $C\left(A_{2}\right)$ are $h_{\alpha}, h_{\beta}$,

$$
\begin{gathered}
c_{1}=Y_{\alpha} X_{\alpha}, \quad c_{2}=Y_{\beta} X_{\beta}, \quad c_{3}=Y_{\alpha+\beta} X_{\alpha+\beta}, \\
c_{4}=Y_{\alpha+\beta} X_{\alpha} X_{\beta}, \quad c_{5}=Y_{\beta} Y_{\alpha} X_{\alpha+\beta} .
\end{gathered}
$$

Each of the $c_{i}$ 's acts on $v_{\lambda}$ to produce a nonzero scalar multiple of $v_{\lambda}$. Define $r, s$, and $z_{i}$ for $1 \leqslant i \leqslant 5$ according to $h_{\alpha} v_{\lambda}=r v_{\lambda}, h_{\beta} v_{\lambda}=s v_{\lambda}$ and $c_{i} v_{\lambda}=z_{i} v_{\lambda}, 1 \leqslant i \leqslant$ 5. Since $M_{\lambda+\alpha+\beta}$ is 1 dimensional, we know that there is some $K \in \mathbf{C}$ such that $X_{\alpha} X_{\beta} v_{\lambda}=K X_{\alpha+\beta} v_{\lambda}$. We show that we can express the $z_{i}$ 's in terms of $r, s$, and $K$ and hence $M$ is uniquely determined by these values.

LEMMA 3.1. Let $r, s$, and $K$ be defined as above, then

$$
\begin{gathered}
z_{1}=(K+r)(K-1), \\
z_{2}=K(K-s-1), \\
z_{3}=(K+r)(K-s-1), \\
z_{4}=z_{5}=K(K+r)(K-s-1) .
\end{gathered}
$$

Moreover, if $\lambda \neq 0$ (i.e. $r \neq 0$ or $s \neq 0$ ) then the mass function $\eta: C\left(A_{2}\right) \rightarrow \mathbf{C}$ defined by $r, s$, and $z_{i}, 1 \leqslant i \leqslant 5$, is in fact determined by $r, s$, and $z_{i}, 1 \leqslant i \leqslant 3$ (i.e. $K$ can be found from these values).

Proof. One can show that the following identities hold in the universal enveloping algebra of $A_{2}$ :

$$
\begin{gathered}
c_{1} c_{2}=c_{2} c_{1}+c_{5}-c_{4}, \\
c_{1} c_{4}=c_{4} c_{1}-c_{2} c_{1}+c_{3} c_{1}-c_{4} h_{\alpha}+c_{3} h_{\alpha}-c_{5}+c_{4}, \\
c_{2} c_{4}=c_{4} c_{2}+c_{2} c_{1}-c_{3} c_{2}-c_{4} h_{\beta}+c_{5}-c_{4} .
\end{gathered}
$$


The definition of $r, s$, and $K$ and these three identities give us respectively

$$
\begin{gathered}
z_{4}=z_{5}, \\
z_{2} z_{1}=z_{3}\left(z_{1}-K r+r\right), \\
z_{2} z_{1}=z_{3}\left(z_{2}+K s\right) .
\end{gathered}
$$

The definition of $K$ produces immediately $z_{4}=K z_{3}$. Also, computing $c_{1} c_{2} v_{\lambda}$ we get

$$
\begin{aligned}
c_{1} c_{2} v_{\lambda} & =Y_{\alpha} X_{\alpha} Y_{\beta} X_{\beta} v_{\lambda}=Y_{\alpha} Y_{\beta} X_{\alpha} X_{\beta} v_{\lambda}=K Y_{\alpha} Y_{\beta} X_{\alpha+\beta} v_{\lambda} \\
& =K\left(Y_{\beta} Y_{\alpha} X_{\alpha+\beta}-Y_{\alpha+\beta} X_{\alpha+\beta}\right) v_{\lambda}=K\left(c_{5}-c_{3}\right) v_{\lambda} \\
& =K\left(c_{4}-c_{3}\right) v_{\lambda}=K(K-1) c_{3} v_{\lambda}
\end{aligned}
$$

or in terms of the $z_{i}$ 's we have

$$
z_{1} z_{2}=K(K-1) z_{3} .
$$

Since none of the $z_{i}$ 's can be zero, (3.11) and (3.12) yield

$$
z_{2}=K(K-s-1) \text {. }
$$

Equating the right-hand sides of (3.10) and (3.11) and using (3.13) produces

$$
z_{1}=(K+r)(K-1) \text {. }
$$

Finally, we obtain the formula for $z_{3}$ from (3.12), (3.13) and (3.14).

The closing statement of this lemma is proved by using (3.2) and (3.4) to solve for $K$, if $s \neq 0$, or by using (3.2) and (3.3) to solve for $K$, if $s=0$ and $r \neq 0$.

Let $M_{\lambda}=\mathbf{C} v_{\lambda}$ be a weight space of the pointed, torsion free $A_{n}$ module $M$. We show

Lemma 3.15. Let $B=\left\{\alpha_{1}, \ldots, \alpha_{n}\right\}$ be a base for the root system $R$ of $A_{n}$. The values $r_{1}, \ldots, r_{n}$ and $K$ defined by $h_{\alpha_{i}} v_{\lambda}=r_{i} v_{\lambda}$ and $X_{\alpha_{1}} X_{\alpha_{2}} v_{\lambda}=K X_{\alpha_{1}+\alpha_{2}} v_{\lambda}$ uniquely determine the action of the basic cycles $c_{i}$ on $v_{\lambda}$.

Proof. According to Lemma 3.1, this lemma is true for $n=2$. Now assume $n>2$. We may assume that this lemma is true for all basic cycles contained in the cycle subalgebra of $A_{n-1}$ determined by the simple roots $\alpha_{1}, \ldots, \alpha_{n-1}$. If the lemma is not true for the basic cycles of $A_{n}$, then according to Lemma 1.8, there must be some $A_{2}$ in $A_{n}$ whose basic cycles are not uniquely determined by $r_{i}, 1 \leqslant i \leqslant n$, and $K$. Evidently, the root system of this $A_{2}$ has a base of the form $\beta=\alpha_{j}+\cdots+\alpha_{k-1}$, $\gamma=\alpha_{k}+\cdots+\alpha_{n}$. These two roots can be incorporated into a base for the root system of $A_{n}$ whose first $n-1$ elements form a base for the root system of $A_{n-1}$, e.g. $-\alpha_{n-1}, \quad-\alpha_{n-2}, \ldots,-\alpha_{k+1}, \quad-\left(\alpha_{k}+\alpha_{k-1}\right), \quad-\alpha_{k-2}, \ldots,-\alpha_{j+1}, \quad-\left(\alpha_{j}+\alpha_{j-1}\right)$, $-\alpha_{j-2}, \ldots,-\alpha_{1}, \alpha_{1}+\cdots+\alpha_{j-1}, \alpha_{j}+\cdots+\alpha_{k-1}, \alpha_{k}+\cdots+\alpha_{n}$. Label the last three roots in this list $\alpha, \beta, \gamma$, respectively.

We now have the problem reduced to a problem concerning $A_{3}$ where $A_{3}$ is the subalgebra determined by $\alpha, \beta, \gamma$. Let $r, s, S$ be defined by $h_{\alpha} v_{\lambda}=r v_{\lambda}, h_{\beta} v_{\lambda}=s v_{\lambda}$ and $X_{\alpha} X_{\beta} v_{\lambda}=S X_{\alpha+\beta} v_{\lambda}$. Clearly $r, s$ are uniquely expressible in terms of the values of the $r_{i}$ 's originally given to us. Also, according to Lemma 3.1, $Y_{\alpha+\beta} X_{\alpha+\beta} v_{\lambda}=$ $(S+r)(S-s-1) v_{\lambda}$ and hence by our assumption on $A_{n-1}, S$ is uniquely determined. 
We introduce some notation which helps us handle the $A_{3}$ case. Whenever $\mu, \nu$, $\mu+\nu$ are roots of $A_{3}$ we define $K(\mu, \nu)$ by $X_{\mu} X_{\nu} v_{\lambda}=K(\mu, \nu) X_{\mu+\nu} v_{\lambda}$. Let

$$
\begin{aligned}
K(\alpha, \beta) & =S, \quad K(\beta, \gamma)=T, \\
K(\alpha+\beta, \gamma) & =U, \quad K(\alpha, \beta+\gamma)=V .
\end{aligned}
$$

Since our inductive assumption implies $S$ is uniquely determined by the $r_{i}$ 's and $K$, it suffices to prove that $T$ is uniquely determined by $r, s$ and $S$. Using relations in the universal enveloping algebra of $A_{3}$, one can readily show that corresponding to the base $-\alpha, \alpha+\beta, \gamma$ the values analogous to (3.16) are

$$
\begin{aligned}
& K(-\alpha, \alpha+\beta)=S+r, \quad K(\alpha+\beta, \gamma)=U, \\
& K(\beta, \gamma)=T, \quad K(-\alpha, \alpha+\beta+\gamma)=V+s .
\end{aligned}
$$

Also, since $T X_{\alpha} X_{\beta+\gamma}=X_{\alpha} X_{\beta} X_{\gamma}=X_{\alpha} X_{\beta+\gamma}+X_{\gamma} X_{\alpha} X_{\beta}$ we have $(T-1) X_{\alpha} X_{\beta+\gamma}=$ $X_{\gamma} X_{\alpha} X_{\beta}$ so that

$$
\begin{aligned}
V(T-1) X_{\alpha+\beta+\gamma} & =(T-1) X_{\alpha} X_{\beta+\gamma}=S X_{\gamma} X_{\alpha+\beta} \\
& =S\left(X_{\alpha+\beta} X_{\gamma}-X_{\alpha+\beta+\gamma}\right)=S(U-1) X_{\alpha+\beta+\gamma}
\end{aligned}
$$

and hence the definitions given by equations (3.16) produce the relationship

$$
S(U-1)=V(T-1) \text {. }
$$

The following series of computations yield linear relationships among $S, T, U$, and $V$.

$$
\begin{aligned}
X_{\alpha} X_{\beta+\gamma} X_{\beta} v_{\lambda} & =\left(X_{\alpha+\beta+\gamma} X_{\beta}+X_{\beta+\gamma} X_{\alpha} X_{\beta}\right) v_{\lambda} \\
& =X_{\alpha+\beta+\gamma} X_{\beta} v_{\lambda}+S X_{\beta+\gamma} X_{\alpha+\beta} v_{\lambda},
\end{aligned}
$$

and

$$
\begin{aligned}
X_{\alpha} X_{\beta+\gamma} X_{\beta} v_{\lambda} & =\left(X_{\alpha+\beta} X_{\beta+\gamma}+X_{\beta} X_{\alpha} X_{\beta+\gamma}\right) v_{\lambda} \\
& =X_{\alpha+\beta} X_{\beta+\gamma} v_{\lambda}+V X_{\alpha+\beta+\gamma} X_{\beta} v_{\lambda} .
\end{aligned}
$$

Subtracting (3.20) from (3.19) and using (3.16), we get

$$
\begin{aligned}
0 & =(S-1) X_{\alpha+\beta} X_{\beta+\gamma} v_{\lambda}+(1-V) X_{\alpha+\beta+\gamma} X_{\beta} v_{\lambda} \\
& =[(S-1) / T] X_{\alpha+\beta} X_{\beta} X_{\gamma} v_{\lambda}+(1-V) X_{\alpha+\beta+\gamma} X_{\beta} v_{\lambda} \\
& =[[U(S-1) / T]+(1-V)] X_{\alpha+\beta+\gamma} X_{\beta} v_{\lambda}
\end{aligned}
$$

which implies

$$
(V-1) T=(S-1) U
$$

When we subtract (3.18) from (3.22) and simplify, we obtain

$$
S-U=V-T \text {. }
$$

The equation analogous to (3.23) derived from (3.17) is

$$
S-T=V-U \text {. }
$$

These last two equations tell us

$$
S=V \text { and } T=U .
$$


As we did in going from (3.16) to (3.17), we choose a new base for our $A_{3}$ subalgebra, namely $\beta, \gamma,-(\alpha+\beta+\gamma)$. Equation (3.24) implies $K(\beta, \gamma)=$ $K(\beta,-(\alpha+\beta))$, by analogy with equation (3.16). By Lemma 3.1, the coefficient $K(\beta,-(\alpha+\beta))$ is uniquely determined by $r, s$ and $S$. This implies that $T$ is uniquely determined by $r, s$, and $S$, since $T=K(\beta, \gamma)$.

The main result of this section is

THEOREM 3.24. If $M$ is a pointed torsion free $A_{n}$ module, then there is an complex $(n+1)$-tuple a such that $M \cong N(a)$.

Proof. Let $\mathbf{C} v_{\lambda}$ be a weight space of $M$ and define $r_{i}, K \in \mathbf{C}$ for $1 \leqslant i \leqslant n$ by setting

$$
h_{\alpha_{i}} v_{\lambda}=r_{i} v_{\lambda} \text { for } 1 \leqslant i \leqslant n
$$

and

$$
X_{\alpha_{1}} X_{\alpha_{2}} v_{\lambda}=K X_{\alpha_{1}+\alpha_{2}} v_{\lambda} .
$$

For any complex $n+1$ tuple $a=\left(a_{1}, \ldots, a_{n+1}\right)$ we have

$$
\begin{gathered}
h_{\alpha_{i}} x^{a}=\left(a_{i}-a_{i+1}\right) x^{a} \quad \text { for } 1 \leqslant i \leqslant n \\
X_{\alpha_{1}} X_{\alpha_{2}} x^{a}=a_{3}\left(a_{2}+1\right) x^{a+\varepsilon_{1}-\varepsilon_{2}} \text { and } X_{\alpha_{1}+\alpha_{2}} x^{a}=a_{3} x^{a+\varepsilon_{1}-\varepsilon_{2}} .
\end{gathered}
$$

Setting $a_{2}=K-1, a_{1}=r_{1}+K-1$ and $a_{i+1}=a_{i}-r_{i}$ for $i=2, \ldots, n$ we have

$$
h_{\alpha_{i}} x^{a}=r_{i} x^{a} \text { for } 1 \leqslant i \leqslant n,
$$

and

$$
X_{\alpha_{1}} X_{\alpha_{2}} x^{a}=K X_{\alpha_{1}+\alpha_{2}} x^{a} .
$$

Therefore by Lemma $3.15 M \cong N(a)$.

4. Pointed torsion free $C_{n}$ modules. In this section we show that every pointed, torsion free $C_{n}$ module is equivalent to one of the modules $M(a)$ constructed in $\$ 2$. Our approach is similar to the method used in $\S 3$ to study $A_{n}$ modules. We first treat the case of $n=2$ and then use this result along with the results of $\S 3$ to establish the general result.

THEOREM 4.1. If $M$ is a pointed, torsion free $C_{2}$ module and $M_{\lambda}=\mathbf{C} v_{\lambda}$ is one of its weight spaces then the action of the cycles of $C_{2}$ on $M_{\lambda}$ is completely determined by $\lambda$.

Proof. Let $B=\{\alpha, \beta\}$ be a base for the root system $R$ of $C_{2}$ and let $\left\{X_{\mu} \mid \mu \in\right.$ $R\} \cup\left\{h_{\alpha}, h_{\beta}\right\}$ be the corresponding standard basis. The elements $h_{\alpha}, h_{\beta}$ together with the basic cycles of $C_{2}$ given by

$$
\begin{array}{lll}
c_{1}=Y_{\beta} X_{\beta}, & c_{5}=Y_{\alpha+\beta} X_{\beta} X_{\alpha}, & c_{9}=Y_{2 \alpha+\beta} X_{\beta} X_{\alpha} X_{\alpha}, \\
c_{2}=Y_{\alpha} X_{\alpha}, & c_{6}=Y_{\alpha} Y_{\beta} X_{\alpha+\beta}, & c_{10}=Y_{\alpha} Y_{\alpha} Y_{2 \alpha+\beta}, \\
c_{3}=Y_{\alpha+\beta} X_{\alpha+\beta}, & c_{7}=Y_{2 \alpha+\beta} X_{\alpha} X_{\alpha+\beta}, & c_{11}=Y_{2 \alpha+\beta} Y_{\beta} X_{\alpha+\beta} X_{\alpha+\beta}, \\
c_{4}=Y_{2 \alpha+\beta} X_{2 \alpha+\beta}, & c_{8}=Y_{\alpha+\beta} Y_{\alpha} X_{2 \alpha+\beta}, & c_{12}=Y_{\alpha+\beta} Y_{\alpha+\beta} X_{\beta} X_{2 \alpha+\beta}
\end{array}
$$

form a generating set for the cycle subalgebra $C\left(C_{2}\right)$ of $C_{2}$. 
Let $0 \neq v_{\lambda} \in M_{\lambda}$ and let $K=K(\beta, \alpha)$ be the coefficient defined by $X_{\beta} X_{\alpha} v_{\lambda}=$ $K X_{\alpha+\beta} v_{\lambda}$. Multiplying this equation on the left by $Y_{\alpha+\beta}, Y_{\alpha} Y_{\beta}, Y_{2 \alpha+\beta} X_{\alpha}$ and $Y_{2 \alpha+\beta} Y_{\beta} X_{\alpha+\beta}$ respectively and letting $c_{i} v_{\lambda}=z_{i} v_{\lambda}$ for $i=1,2, \ldots, 12$ we obtain the following relations:

$$
\begin{array}{ll}
z_{5}=K z_{3}, & (K+2) z_{6}=z_{1} z_{2}, \\
z_{9}=(K-2) z_{7}+2 z_{4}, & K z_{11}=z_{1}\left(z_{7}-z_{4}\right) .
\end{array}
$$

The following identities hold in the universal enveloping algebra of $C_{2}$ :

(4.3) $\quad\left[c_{1}, c_{2}\right]=2 c_{5}-2 c_{6}$

(4.4) $\left[c_{2}, c_{4}\right]=2 c_{7}-2 c_{8}$,

(4.5) $\left[c_{1}, c_{7}\right]=-2 c_{11}+2 c_{9}+8 c_{7}-4 c_{4}$,

(4.6) $\left[c_{4}, c_{5}\right]=2 c_{12}-2 c_{9}-4 c_{8}-4 c_{7}+4 c_{4}$,

(4.7) $\left[c_{4}, c_{6}\right]=-2 c_{11}+2 c_{10}+4 c_{8}+4 c_{7}-4 c_{4}$,

(4.8) $\left[c_{1}, c_{5}\right]=-2 c_{2} c_{1}-2 c_{1} c_{3}+4 h_{\beta}\left(c_{5}+2 c_{3}\right)$,

(4.9) $\left[c_{4}, c_{11}\right]=-4\left(h_{\alpha}+h_{\beta}\right) c_{11}+4 c_{4} c_{6}+8 c_{4} c_{3}-2 c_{4} c_{1}$,

(4.10) $\left[c_{2}, c_{7}\right]=-2 c_{2} c_{3}-2 c_{6}+c_{2} c_{4}-h_{\alpha}\left(c_{7}-c_{4}\right)+c_{9}+2 c_{8}-2 c_{4}$,

(4.11) $\left[c_{2}, c_{5}\right]=-c_{2} c_{1}+2 c_{2} c_{3}-c_{9}-2 c_{8}-\left(h_{\alpha}+2\right) c_{5}+2 c_{4}+4 c_{6}$.

Define $r, s \in \mathbf{C}$ by $h_{\alpha} v_{\lambda}=r v_{\lambda}$ and $h_{\beta} v_{\lambda}=s v_{\lambda}$. Applying the elements represented by (4.3) through (4.11) to $v_{\lambda}$, we get a series of relations (4.3)' through (4.11)' respectively, involving $r, s$ and the $z_{i}$ 's. Using equations (4.3)' and (4.4)', we obtain

$$
\begin{aligned}
& z_{5}=z_{6}, \quad \text { and } \\
& z_{7}=z_{8} .
\end{aligned}
$$

Adding (4.5)' to (4.6)' and using (4.13) gives us

$$
z_{11}=z_{12} \text {. }
$$

Similarly using $(4.6)^{\prime},(4.7)^{\prime}$ and $(4.14)$, we find

$$
z_{9}=z_{10} .
$$

Now we use (4.8)' to express $z_{1}$ in terms of $K$ and $s$.

$$
\begin{aligned}
0 & =-2 z_{1} z_{2}-2 z_{1} z_{3}+4 s\left(z_{5}+2 z_{3}\right) \quad \text { by }(4.8)^{\prime} \\
& =-2(K+2) z_{6}-2 z_{1} z_{3}+(4 s K+8 s) z_{3} \quad \text { by }(4.2) \\
& =2 z_{3}\left[-(K+2) K-z_{1}+2 s(K+2)\right] \quad \text { by }(4.2) \text { and }(4.12)
\end{aligned}
$$

and hence

$$
z_{1}=-(K+2)(K-2 s) .
$$

We can express $z_{7}$ in terms of $z_{4}, K$ and $s$ as follows

$$
\begin{aligned}
0 & =-2 z_{11}+2 z_{9}+8 z_{7}-4 z_{4} \quad \text { by }(4.5)^{\prime} \\
& =-2 z_{11}+2\left[(K-2) z_{7}+2 z_{4}\right]+8 z_{7}-4 z_{4} \quad \text { by }(4.2),
\end{aligned}
$$


which simplifies after multiplication by $K / 2$ to

$$
\begin{aligned}
0 & =-K z_{11}+K(K+2) z_{7} \\
& =[K(K+2)+(K+2)(K-2 s)] z_{7}-(K+2)(K-2 s) z_{4}
\end{aligned}
$$

with the last equality following from (4.2)' and (4.16)' and simplifying to give

$$
z_{7}=[(K-2 s) / 2(K-s)] z_{4} .
$$

We use this relationship to express $z_{11}$ in terms of $s, K$ and $z_{4}$ by modifying (4.6)

$$
\begin{aligned}
0 & =2 z_{12}-2 z_{9}-4 z_{8}-4 z_{7}+4 z_{4} \text { by }(4.6)^{\prime} \\
& =2 z_{11}-2(K-2) z_{7}-4 z_{4}-4 z_{8}-4 z_{7}+4 z_{4}, \quad \text { by }(4.2) \text { and }(4.14) \text {, or } \\
0 & =z_{11}-(K+2) z_{7}, \quad \text { by }(4.13) .
\end{aligned}
$$

This expression and (4.17) give us

$$
z_{11}=[(K+2)(K-2 s) / 2(K-s)] z_{4} .
$$

Substitute this value as well as the one given by (4.17) into (4.6)' to get

$$
\begin{aligned}
z_{9} & =[(K+2)(K-2 s) / 2(K-s)-4(K-2 s) / 2(K-s)+2] z_{4} \\
& =[K(K-2 s+2) / 2(K-s)] z_{4} .
\end{aligned}
$$

We see now that determining $z_{4}$ determines several of the $z_{i}$ values we need. Before trying to find $z_{4}$, we first find $z_{3}$. From (4.9)' we get

$$
\begin{aligned}
z_{6} & =(r+s) z_{11} / z_{4}-2 z_{3}+z_{1} / 2 \\
& =(r+s)(K+2)(K-2 s) / 2(K-s)-(K+2)(K-2 s) / 2-2 z_{3} \\
& =-(K+2)(K-2 s)(K-2 s-r) / 2(K-s)-2 z_{3} \quad \text { by }(4.16) \text { and }(4.18) \\
& =K z_{3} \quad \text { by }(4.2)
\end{aligned}
$$

and hence

$$
\begin{aligned}
& z_{3}=-(K-2 s)(K-2 s-r) / 2(K-s) \text { and } \\
& z_{6}=-K(K-2 s)(K-2 s-r) / 2(K-s) .
\end{aligned}
$$

Moreover, we can now use (4.2) to determine $z_{2}$.

$$
z_{2}=(K+2) z_{6} / z_{1}=K(K-2 s-r) / 2(K-s) \text { by (4.21). }
$$

Our remaining unused primed equations (4.10) $)^{\prime}$ and (4.11) $)^{\prime}$ allow us to express $z_{4}$ and $K$ in terms of $r$ and $s$. First add these equations together to get

$$
\begin{aligned}
0= & -z_{2} z_{1}-r z_{5}+z_{2} z_{4}+r z_{4}-r z_{7}-2 z_{5}+2 z_{6} \\
= & {[K(K-2 s-r)(K+2)(K-2 s)+r K(K-2 s)(K-2 s-r)] / 2(K-s) } \\
& +[[K(K-2 s-r)+r(2(K-s))-r(K-2 s)] / 2(K-s)] z_{4}
\end{aligned}
$$

which implies

$$
z_{4}=-(K-2 s-r)(K+2+r)
$$


Now multiply equation $(4.11)^{\prime}$ by $2(K-s)^{2}$ and use the values we have obtained for the $z_{i}$ 's as follows:

$$
\begin{aligned}
0= & 2(K-s)^{2}\left[-z_{2} z_{1}+2 z_{2} z_{3}-z_{9}-2 z_{8}-(r-2) z_{6}+2 z_{4}\right] \\
= & K(K-2 s)(K-2 s-r) \\
& \cdot[(K-s)(K+2)-(K-2 s-r)+(r-2)(K-s)] \\
& -(K-s)[K(K-2 s+2)+2(K-2 s)-4(K-s)] z_{4} \\
= & K(K-2 s)(K-2 s-r)[(K-s)(K+r)-(K-2 s-r)] \\
& +K(K-s)(K-2 s)(K-2 s-r)(K+2+r), \text { or } \\
0= & 2(K-s)[K+r+1]-(K-2 s-r)=2[K-(2 s-1) / 2][K+r] .
\end{aligned}
$$

Therefore, $K=(2 s-1) / 2$, or $K=-r$. To see that the second possibility cannot occur, note that if $K=-r$ then $z_{2}=K$ and we arrive at a contradiction by computing

$$
X_{\alpha} Y_{\alpha} v_{\lambda}=\left(h_{\alpha}+Y_{\alpha} X_{\alpha}\right) v_{\lambda}=(r+K) v_{\lambda}=0 \text {. }
$$

Equation (4.26) implies that either $X_{\alpha}$ or $Y_{\alpha}$ is not torsion free contrary to assumption.

THEOREM 4.27. If $M$ is a pointed, torsion free $C_{n}$ module then there exists a complex $n$-tuple a such that $M \cong M(a)$.

Proof. Let $\lambda$ be a weight function of $M$ such that $\lambda\left(h_{\alpha}\right) \neq 0$ for all roots $\alpha$ of $C_{n}$. Let $v_{\lambda}$ be a nonzero vector in $M_{\lambda}$. If $a=\left(a_{1}, \ldots, a_{n}\right)$ is the unique complex $n$ tuple such that $h_{\alpha_{i}} v_{\lambda}=\left(a_{i}-a_{i+1}\right) v_{\lambda}$ for $i=1, \ldots, n-1$ and $h_{\alpha_{n}} v_{\lambda}=\left(\left(a_{n}+1\right) / 2\right) v_{\lambda}$, then $M(a)$ is a pointed, torsion free $C_{n}$ module admitting $\lambda$ as a weight function. In order to verify that $M \cong M(a)$, it suffices by Theorem 1.9 to show that the action of the $C_{n}$ basic cycles of degree less than or equal to 3 on $M_{\lambda}$ are completely determined by $\lambda$.

Since each basic cycle $c$ of degree 2 belongs to a $C_{2}$ subalgebra of $C_{n}$, Lemma 4.1 implies that the value of $c v_{\lambda}$ is uniquely determined by $\lambda$. Any basic cycle $c^{\prime}$ of degree 3 belongs to either a $C_{2}$ or an $A_{2}$ subalgebra of $C_{n}$. If $c^{\prime}$ belongs to a $C_{2}$ subalgebra, Lemma 4.1 again implies that $c^{\prime} v_{\lambda}$ is determined by $\lambda$. If $c^{\prime}$ belongs to an $A_{2}$ subalgebra, Lemma 3.13 states that $c^{\prime} v_{\lambda}$ is determined by the values of the basic cycles of degree less than or equal to 2 . However from the argument above, these values are uniquely determined by $\lambda$ and hence so is $c^{\prime} v_{\lambda}$.

5. General construction of simple pointed modules. Fernando [8] has shown that all simple $(L, H)$ weight modules are equivalent to simple quotients of modules induced from torsion free modules $M^{u^{+}}$of certain reductive subalgebras $(g, H)$ of $(L, H)$. The problem then is to determine all possible torsion free modules. In $\S \S 3$ and 4 we have constructed all pointed torsion free modules. One can use these torsion free modules and apply parabolic induction as presented by Fernando to 
construct pointed modules. We can conclude from the results below that in fact this process yields all pointed modules. However, in this section we present an alternate construction of these modules which realizes all pointed modules as simple quotients of $U(L)$.

We first require some general results concerning the classification of the roots of $L$ as torsion free or locally nilpotent for a given $(L, H)$ weight module $M$. Fernando [8] has shown that the set of torsion free roots $T(M)$ is a "convex" subset of $R$, i.e., all roots which are positive rational linear combinations of roots in $T(M)$ are contained in $T(M)$. We now use this result to prove

LEMma 5.1. $N=\{\mu \in R \mid \pm \mu \notin T(M)\}$ is a root subsystem of $R$.

Proof. We first show that if $\bar{R}$ is any connected, rank 2 subsystem of $R$ with $\bar{R} \cap N$ containing more than two linearly independent roots then $\bar{R} \subseteq N$. We proceed by contradiction assuming that $\bar{R} \nsubseteq N$-i.e. there exists at least one torsion free root in $\bar{R}$.

Since $\bar{R}$ is of rank 2 and contains both torsion free and locally nilpotent roots we may select two roots $\mu, \nu \in \bar{R}$ such that $\mu$ is torsion free, $\nu$ is locally nilpotent and no positive linear combination of $\mu$ and $\nu$ are roots. Clearly $\{\mu,-\nu\}$ forms a base of $\bar{R}$. If $-\nu$ is torsion free then by the convexity of $T(M)$ all roots in $\bar{R}$ which are positive with respect to $\{\mu,-\nu\}$ are torsion free and hence $\bar{R} \cap N=\varnothing$ contrary to assumption. We may therefore assume that $-\nu$ is locally nilpotent.

Since $\nu$ is locally nilpotent there exists a nonzero vector $v \in M$ such that $X_{\nu} v=0$. Since $-\nu$ is locally nilpotent there exists a nonnegative integer $j$ such that $h_{\nu} v=j v$ where $h_{\nu}$ denotes the element of the Cartan subalgebra which is dual to $\nu$ with respect to the Killing form. Then for any nonnegative integer $m$ we have

(i) $X_{\mu}^{m} v \neq 0$ since $\mu$ is torsion free,

(ii) $X_{\nu} X_{\mu}^{m} v=0$ since $\left[X_{\nu}, X_{\mu}\right]=0$,

(iii) $h_{\nu} X_{\mu}^{m} v=(m\langle\mu, \nu\rangle+j) X_{\mu}^{m} v$ where $\langle\cdot, \cdot\rangle$ denotes the Killing form on $H^{*}$. It follows that $X_{-\nu}^{p} X_{\mu}^{m} v \neq 0$ for all integers $p$ with $0 \leqslant p \leqslant m\langle\mu, \nu\rangle+j$.

For any root $r \mu+s(-\nu) \in \bar{R}$ with $r \geqslant 1$ we have that $\langle r \mu+s(-\nu),-\nu\rangle \leqslant s$ which implies that $s \leqslant r\langle\mu, \nu\rangle$. Therefore, for all nonnegative integers $k$, sk $r k\langle\mu, \nu\rangle+j$ and hence $X_{-\nu}^{s k} X_{\mu}^{r k} v \neq 0$. Then by Theorem 1.5 all roots $r \mu+s(-\nu)$ with $r \geqslant 1$ are torsion free and hence $\bar{R} \cap N=\{ \pm \nu\}$ contrary to assumption. It follows then that $\bar{R} \subseteq N$ as required.

Since $N$ is a subset of a root system $R$, in order to show that $N$ is a root subsystem it suffices to show that $\alpha, \beta \in N$ implies $\sigma_{\alpha}(\beta) \in N$ where $\sigma_{\alpha}$ is the Weyl reflection in the hyperplane perpendicular to $\alpha$. If $\alpha= \pm \beta$ the $\sigma_{\alpha}(\beta)=-\beta \in N$. If $\langle\alpha, \beta\rangle=0$, the $\sigma_{\alpha}(\beta)=\beta \in N$. Finally if $\langle\alpha, \beta\rangle \neq 0$ and $\alpha \neq \pm \beta$, then $\{\alpha, \beta\}$ spans a connected rank 2 subsystem $\bar{R}$ of $R$ with $\{ \pm \alpha, \pm \beta\} \subseteq \bar{R}$ and hence by our previous argument $\sigma_{\alpha}(\beta) \in \bar{R} \subseteq N$ as required.

THEOREM 5.2. Let $\lambda$ be a weight of $M^{u^{+}}$and $\gamma$ be a weight of $M$. Then $\operatorname{dim} M_{\gamma} \geqslant \operatorname{dim} M_{\lambda}$. 
Proof. Let $0 \neq v_{\lambda} \in M_{\lambda}$ and $0 \neq v_{\gamma} \in M_{\gamma}$ and assume $\operatorname{dim} M_{\lambda}>\operatorname{dim} M_{\gamma}$. We may assume further that $\lambda$ and $\gamma$ are related by $\gamma=\lambda+\sum k_{i} \alpha_{i}$ where $\alpha_{i} \in B$ and $k_{i} \geqslant 0$. Moreover, we may take $\lambda$ and $\gamma$ so that $\sum k_{i}$ is minimal among all such pairs.

By the irreducibility of $M$ and the Poincaré-Birkhoff-Witt Theorem, we can redefine $v_{\gamma}$ so that it is the image of $v_{\lambda}$ under the action of a monomial in the $\sum k_{i} \alpha_{i}$ weight space of $U(L)$ and further that this monomial involves no elements of $H$ and no torsion free root vectors. Lemma 5.1 implies that all roots in the root system spanned by $\left\{\alpha_{i} \mid k_{i} \neq 0\right\}$ are locally nilpotent.

The subset $B_{1}=\left\{\alpha_{i} \mid k_{i} \neq 0\right\}$ of $B$ is a base for a semisimple subalgebra $L_{1}$ of $L$. The weight vectors of $M_{\lambda}$ are lowest weight vectors relative to $B_{1}$ in the $L_{1}$-module $M_{1}=U\left(L_{1}\right) M_{\lambda}$. Our result then follows from the theory of finite-dimensional modules once we have shown that $M_{1}$ is finite dimensional.

Let $X_{1}^{e_{1}} \cdots X_{k}^{e_{k}}$ be a typical monomial in a Poincaré-Birkhoff-Witt basis of $U\left(L_{1}\right)$. (Here the $X_{i}$ 's denote root vectors in $L_{1}$.) Then

$$
M_{1}=\text { lin. } \operatorname{span}\left\{X_{1}^{e_{1}} \cdots X_{k}^{e_{k}} M_{\lambda} \mid e_{i}{ }^{\prime} s \text { are nonnegative integers }\right\} .
$$

If $S_{k-i}=$ lin. $\operatorname{span}\left\{X_{k-i}^{e_{k-i}} \cdots X_{k}^{e_{k}} M_{\lambda} \mid e_{j}\right.$ 's are nonnegative integers $\}$ is finite dimensional then since $X_{k-i-1}$ is locally nilpotent there is some $m$ such that $X_{k-i-1} m_{S_{i}}=0$ and hence $S_{k-i-1}$ is finite dimensional. It now follows, since $M_{\lambda}$ is finite dimensional, $M_{1}=S_{k-1}$ is also finite dimensional.

COROLlary 5.3. If $M$ is pointed then every weight space of $M^{u^{+}}$is 1 dimensional.

TheORem 5.4 [13, TheORem 6]. Let L be a simple Lie algebra with Cartan subalgebra $H$. Let $B$ be a base of the root system $R$ of $L$ with respect to $H$. Let $\left\{B_{i} \mid i=1, \ldots, k\right\}$ be mutually orthogonal components of $B$ and set $L_{i}$ equal to the subalgebra of $L$ associated with the root subsystem $R_{i}$ of $R$ generated by $B_{i}$. Then the cycle subalgebra $C(L)$ can be written as a vector space direct sum

$$
C(L)=U(H) C\left(L_{1}\right) \cdots C\left(L_{K}\right) \oplus C^{\prime}
$$

where $C^{\prime}$ is the linear span of all $P B W$ basis monomials of $C(L)$ involving at least one root vector not in $\cup R_{i}$. The subspace $C^{\prime}$ is an ideal in $C(L)$ and moreover if $\eta_{i}$ : $C\left(L_{i}\right) \rightarrow \mathbf{C}$ are mass functions there exists a mass function $\eta: C(L) \rightarrow \mathbf{C}$ such that $\eta\left|C\left(L_{i}\right)=\eta_{i}, \eta\right| C^{\prime}=0$ and $\eta$ may be defined arbitrarily on the basis elements $H_{\mu}$ where $\mu \notin \cup R_{i}$.

We now indicate how the construction and classification of all torsion free modules for algebras of types $A$ and $C$ lead to an alternate construction and classification of all pointed $L$ modules. We first use the foregoing results to observe that every pointed $L$ module admits (and hence by Theorem 1.8 is determined by) a mass function of a certain type. In fact, if $M$ is a pointed $L$ module then by Corollary 5.3 we may select a 1-dimensional weight space $M_{\lambda}$ contained in $M^{u^{+}}$. By Theorem 1.3 there exists a base $B$ of the root system $R$ of $L$ such that the symmetric part of $T(M)$ intersects $B$ in mutually orthogonal components $\left\{B_{i} \mid i=1, \ldots, k\right\}$ where each $B_{i}$ is a base of the root system $R_{i}$ of a simple Lie subalgebra $L_{i}$ of type 
$A$ or $C$. Then the $L_{i}$ submodule $M_{i}$ of $M$ generated by $M_{\lambda}$ is a torsion free $L_{i}$ module. Therefore the mass function $\eta: C(L) \rightarrow \mathrm{C}$ of the pointed $L$ module $M$ associated with the 1-dimensional weight space $M_{\lambda}$ has the following properties:

(i) $\eta \mid H=\lambda$,

(ii) $\eta \mid C\left(L_{i}\right)=$ a mass function of a pointed torsion free $L_{i}$ module, and

(iii) $\eta \mid C^{\prime}=0$.

Conversely then, using the classification of all pointed torsion free modules (and hence all mass functions of pointed torsion free modules) and applying Theorem 5.4 we can construct all mass functions $\eta: C(L) \rightarrow \mathrm{C}$ having properties (i)-(iii). The $L$ module $U(L) / I_{\eta}$ associated to each such mass function $\eta$ where $I_{\eta}$ denotes the unique left ideal of $U(L)$ containing the kernel of $\eta$ is then a pointed $L$ module. Moreover the collection of all such $L$ modules yields all pointed $L$ modules.

\section{REFERENCES}

1. D. Arnal and G. Pinczon, Sur certaines de l'algèbre de Lie sl(2), C. R. Acad. Sci. Paris Ser. A-B 272 (1971), 1369-1372.

2. R. Block, The irreducible representations of the Lie algebra $\mathrm{sl}(2)$ and of the Weyl algebra, Adv. in Math. 39 (1981), 69-110.

3. I. Bouwer, Standard representations of simple Lie algebras, Canad. J. Math. 20 (1968), 344-361.

4. D. J. Britten and F. W. Lemire, Irreducible representations of $A_{n}$ with a 1-dimensional weight space, Trans. Amer. Math. Soc. 273 (1982), 509-540.

5.

6. J. Dixmier, Algèbres enveloppantes, Gauther-Villars, Paris, 1974.

7. E. Dynkin, Semisimple subalgebras of semisimple Lie algebras, Amer. Math. Soc. Transl. (2) 6 (1957), 111-243.

8. Suren L. Fernando, Simple weight modules of complex reductive Lie algebras, $\mathrm{Ph}$. D. Thesis, Univ. of Wisconsin, 1983.

9. J. Humphreys, Introduction to Lie algebras and representation theory, Graduate Texts in Math., No. 9, Springer-Verlag, New York, 1972.

10. F. Lemire, Irreducible representations of a simple Lie algebra admitting a one-dimensional weight space, Proc. Amer. Math. Soc. 19 (1968), 1161-1164.

11. , Note on weight spaces of irreducible linear representations, Canad. Math. Bull. 11 (1968), 399-404.

12. Weight spaces and irreducible representations of simple Lie algebras, Proc. Amer. Math. Soc. 22 (1969), 192-197.

13. , One dimensional representations of the cycle subalgebra of a semisimple Lie algebra, Canad.

Math. Bull. 13 (1970), 463-467.

14. __ An irreducible representation of sl(2), Canad. Math. Bull. 17 (1974), 63-64.

15. $\ldots$ A new family of irreducible representations of $A_{n}$, Canad. Math. Bull. 18 (1975), 543-546.

16. F. Lemire and M. Pap, H-finite representations of simple Lie algebras, Canad. J. Math. 30 (1979), 1084-1106.

17. A. van den Hombergh, Sur des suites de racines dont la sommes des termes est nulle, Bull. Soc. Math. France 102 (1974), 353-364.

Department of Mathematics and Statistics, University of Windsor, Windsor, Ontario, CANADA N9B 3P4 